\title{
Signals for new spin-1 resonances in electroweak gauge boson pair production at the LHC
}

\author{
A. Alves, ${ }^{1, *}$ O. J.P. Éboli, ${ }^{2, \dagger}$ D. Gonçalves Netto, ${ }^{2,}$ M. C. Gonzalez-Garcia, ${ }^{3, \S}$ and J. K. Mizukoshi ${ }^{4, \|}$ \\ ${ }^{1}$ Instituto de Física Teórica, Universidade Estadual Paulista, São Paulo - SP, Brazil. \\ ${ }^{2}$ Instituto de Física, Universidade de São Paulo, São Paulo - SP, Brazil. \\ ${ }^{3}$ C.N. Yang Institute for Theoretical Physics, SUNY at Stony Brook, Stony Brook, New York 11794-3840, USA, \\ Institució Catalana de Recerca i Estudis Avançats (ICREA), Departament d'Estructura i Constituents de la Matèria, \\ Universitat de Barcelona, 647 Diagonal, E-08028 Barcelona, Spain \\ ${ }^{4}$ Centro de Ciências Naturais e Humanas, Universidade Federal do ABC, Santo André - SP, Brazil.
}

(Received 3 August 2009; published 23 October 2009)

\begin{abstract}
The mechanism of electroweak symmetry breaking (EWSB) will be directly scrutinized soon at the CERN Large Hadron Collider. We analyze the LHC potential to look for new vector bosons associated with the EWSB sector, presenting a possible model independent approach to search for these new spin-1 resonances. We show that the analyses of the processes $p p \rightarrow \ell^{+} \ell^{\prime-} \mathscr{E}_{T}, \ell^{ \pm} j j \mathscr{E}_{T}, \ell^{\prime \pm} \ell^{+} \ell^{-} \mathscr{E}_{T}, \ell^{ \pm} j j \mathscr{E}_{T}$, and $\ell^{+} \ell^{-} j j$ (with $\ell, \ell^{\prime}=e$ or $\mu$ and $j=$ jet) have a large reach at the LHC and can lead to the discovery or exclusion of many EWSB scenarios such as Higgsless models.
\end{abstract}

DOI: 10.1103/PhysRevD.80.073011

PACS numbers: 14.70.Pw

\section{INTRODUCTION}

The CERN Large Hadron Collider is about to start probing directly the $\mathrm{TeV}$ scale with the study of the electroweak symmetry breaking mechanism being at center stage. In order to respect unitarity in the weak gauge boson scattering $W_{L}^{+} W_{L}^{-} \rightarrow W_{L}^{+} W_{L}^{-}$there must be a contribution of the EWSB at the TeV scale [1], well within the LHC reach. In the SM the growth of the scattering amplitude of this process is cutoff by the presence of the Higgs boson, however, the simplest one doublet Higgs sector is just one of a myriad of possibilities for the symmetry breaking sector of the electroweak interactions.

One appealing possibility is that the electroweak symmetry breaking is caused by a new strongly interacting sector [2]. Although simple scaled up QCD models are ruled out by precision measurements and flavor changing neutral current constraints, it is possible to build viable models of dynamical electroweak symmetry breaking [3]. A common feature of many of these models is the appearance of new spin-1 states that unitarize the weak gauge boson scattering.

Recently scenarios presenting extra dimensions have received a lot of attention, allowing the construction of Higgsless models where unitarity restoration takes place through the exchange of an infinite tower of spin-1 KaluzaKlein (KK) excitations of the known electroweak gauge bosons [4]. In this class of models [5] unitarity constraints give rise to sum rules depending on the couplings and masses of the KK excitations that imply that the first KK excitation should be observable at the LHC, while higher KK modes are probably beyond the LHC reach [6].

\footnotetext{
*aalves@fma.if.usp.br

†eboli@fma.if.usp.br

*dorival@fma.if.usp.br

§concha@insti.physics.sunysb.edu

"mizuka@ufabc.edu.br
}

A common feature of many EWSB scenarios, as the ones above mentioned, is the existence of new vector resonances, $Z^{\prime}$ and $W^{\prime}$, that couple to $W^{+} W^{-}$and $W^{ \pm} Z$ pairs, respectively. But, generically, their properties, such as mass, width, and couplings to SM fermions, are model dependent. In this respect, the most model independent channel for detection of such spin-1 resonances would be the observation of their contribution to SM gauge boson fusion (WBF) which only involves their couplings to electroweak gauge bosons. At the LHC this would correspond to the $Z^{\prime}$ exchange contribution to $W^{+} W^{-} \rightarrow W^{+} W^{-}$, such as, for example, in the process $p p \rightarrow j j \ell^{+} \ell^{\prime-} \mathscr{E}_{T}$. Unfortunately, that signal is unobservable at LHC even with increased luminosity [6,7]. On the other hand, $W^{\prime}$ can be observed in the WBF $W^{ \pm} Z \rightarrow W^{ \pm} Z$ elastic scattering $[6,7]$.

The new spin-1 states can also be directly produced in $p p$ collisions via their couplings to light quarks. In this case, in order to determine that such new vector bosons are indeed associated with EWSB one can study processes in which the new spin-1 decays into electroweak gauge boson pairs, i.e.

$$
\begin{gathered}
p p \rightarrow Z^{\prime} \rightarrow W^{+} W^{-} \rightarrow \ell^{+} \ell^{\prime-} \mathscr{E}_{T} \text { and } \ell^{ \pm} j j \mathscr{E}_{T} \\
p p \rightarrow W^{\prime} \rightarrow W^{ \pm} Z \rightarrow \ell^{\prime \pm} \ell^{+} \ell^{-} \mathscr{E}_{T}, \\
\ell^{ \pm} j j \mathscr{E}_{T} \text { and } \ell^{+} \ell^{-} j j
\end{gathered}
$$

where $\ell$ and $\ell^{\prime}$ stand for electrons and muons and $j$ for jets.

In this work we present a model independent analysis of the observability of these signals. We express our results as function of the relevant spin-1 boson effective couplings, mass and width. For $Z^{\prime}$ states these are the most sensitive channels which can give information on its connection to EWSB. For $W^{\prime}$ they complement its observation in WBF and provide further information on the $W^{\prime}$ properties. The outline of this work is as follows: in Sec. II we present the 
framework of our analysis and the underlying assumptions in our model independent approach. In Sec. III we describe the differentiating characteristics between the signal and SM backgrounds contributing to the processes (1) and (2) and quantify those after imposing the corresponding cuts to optimize the signal to background ratio. Finally, in Sec. IV we present and discuss the obtainable sensitivities in the different channels as a function of the relevant parameters.

\section{FRAMEWORK OF OUR ANALYSES}

From the phenomenological point of view, the study of processes (1) and (2) requires the knowledge of the couplings of the new spin-1 states to the light quarks and to electroweak vector boson pairs, in addition to their masses and widths.

In this work, for the sake of concreteness, we assume that the couplings of the $Z^{\prime}$ and the $W^{\prime}$ to the light quarks and to gauge bosons have the same Lorentz structure as those of the SM, as it is the case for Higgsless models, but with rescaled strength. In Higgsless models, the unitarity bound in $W^{+} W^{-} \rightarrow W^{+} W^{-}$is saturated by the exchange of the first Kaluza-Klein excitation (or $Z^{\prime}$ ) if its couplings to electroweak gauge bosons is given by [6]

$$
g_{Z^{\prime} W W \max }=g_{Z W W} \frac{M_{Z}}{\sqrt{3} M_{Z^{\prime}}}
$$

for a given $Z^{\prime}$ mass. Correspondingly, the saturation of unitarity in the elastic scattering $W^{ \pm} Z \rightarrow W^{ \pm} Z$ leads to the constraint [6]

$$
g_{W^{\prime} W Z \max }=g_{W W Z} \frac{M_{Z}^{2}}{\sqrt{3} M_{W^{\prime}} M_{W}} .
$$

These constraints imply an upper bound on the decay width of the KK-bosons into SM gauge bosons. In what follows we use $g_{W^{\prime} W Z \max }$ and $g_{Z^{\prime} W W \max }$ simply as convenient normalizations for the coupling of the spin-1 resonance to SM gauge bosons. Moreover, in a generic model, the new spin-1 states have further couplings to other particles, e.g. $b$ or $t$ quarks, that contribute to their width. Therefore, in this work we treat the $Z^{\prime}$ and $W^{\prime}$ widths as free parameters. With these assumptions it is possible to make a model independent analysis of the observability of the relevant processes.

Within this framework the cross section for the processes (1) and (2) can be written in full generality as

$$
\begin{aligned}
\sigma_{\text {tot }}= & \sigma_{\mathrm{SM}}+\left(\frac{g_{V^{\prime} q \bar{q}}}{g_{V q \bar{q}}} \frac{g_{V^{\prime} W V}}{g_{V^{\prime} W V \max }}\right) \sigma_{\mathrm{int}}\left(M_{V^{\prime}}, \Gamma_{V^{\prime}}\right) \\
& +\left(\frac{g_{V^{\prime} q \bar{q}}}{g_{V q \bar{q}}} \frac{g_{V^{\prime} W V}}{g_{V^{\prime} W V \max }}\right)^{2} \sigma_{V^{\prime}}\left(M_{V^{\prime}}, \Gamma_{V^{\prime}}\right)
\end{aligned}
$$

where for processes (1) $V^{\prime}=Z^{\prime}, g_{V^{\prime} W V} \equiv g_{Z^{\prime} W W}$, and $g_{V q \bar{q}} \equiv g_{Z q \bar{q}}=g / c_{W}$. For processes (2) $V^{\prime}=W^{\prime}$, $g_{V^{\prime} W V} \equiv g_{W^{\prime} W Z}$, and $g_{V q \bar{q}} \equiv g_{W q \bar{q}^{\prime}}=g / \sqrt{2}$. Here $g$ is the $S U(2)_{L}$ coupling constant and $c_{W}$ the cosine of the weak mixing angle. We notice that the final state $p p \rightarrow$ $\ell^{ \pm} j j \not E_{T}$ can receive contributions from both $Z^{\prime}$ and $W^{\prime}$ intermediate states. When studying this channel we consider the sensitivity to each of the contributions separately.

In this approach, for each final state the analysis depends on three parameters: the mass of the new spin-1 gauge boson, $M_{V^{\prime}}$, its width, $\Gamma_{V^{\prime}}$, and the product of its couplings to light quarks and to SM gauge bosons, $g_{V^{\prime} q \bar{q}} g_{V^{\prime} V V}$. These parameters are only subject to the constraint that for a given value of product of the couplings of the new spin- 1 boson and of its mass, there is lower bound on its width, since

$$
\begin{gathered}
\Gamma_{Z^{\prime}} \geq \sum_{q=u, d} \Gamma\left(Z^{\prime} \rightarrow q \bar{q}\right)+\Gamma\left(Z^{\prime} \rightarrow W^{+} W^{-}\right) \\
\Gamma_{W^{\prime+}} \geq \Gamma\left(W^{\prime+} \rightarrow u \bar{d}\right)+\Gamma\left(W^{\prime+} \rightarrow W^{+} Z\right) .
\end{gathered}
$$

Using the values the partial widths (all in $\mathrm{GeV}$ ):

$$
\begin{aligned}
\Gamma\left(Z^{\prime} \rightarrow u \bar{u}\right) & =0.3\left(\frac{M_{Z^{\prime}}}{M_{Z}}\right)\left(\frac{g_{Z^{\prime} q \bar{q}}}{g_{Z q \bar{q}}}\right)^{2} \\
\Gamma\left(Z^{\prime} \rightarrow d \bar{d}\right) & =0.38\left(\frac{M_{Z^{\prime}}}{M_{Z}}\right)\left(\frac{g_{Z^{\prime} q \bar{q}}}{g_{Z q \bar{q}}}\right)^{2} \\
\Gamma\left(Z^{\prime} \rightarrow W^{+} W^{-}\right) & =0.028\left(\frac{M_{Z^{\prime}}}{M_{Z}}\right)^{3}\left(\frac{g_{Z^{\prime} W W}}{g_{Z^{\prime} W W \max }}\right)^{2} \\
\Gamma\left(W^{\prime+} \rightarrow q^{\prime} \bar{q}\right) & =0.68\left(\frac{M_{W^{\prime}}}{M_{W}}\right)\left(\frac{g_{W^{\prime} q \bar{q}}}{g_{W q \bar{q}}}\right)^{2} \\
\Gamma\left(W^{\prime+} \rightarrow W^{+} Z\right) & =0.019\left(\frac{M_{W^{\prime}}}{M_{W}}\right)^{3}\left(\frac{g_{W^{\prime} W Z}}{g_{W^{\prime} W Z \max }}\right)^{2},
\end{aligned}
$$

it is possible to show that the minimum $V^{\prime}$ decay widths (in $\mathrm{GeV})$ are:

$$
\begin{gathered}
\Gamma_{Z^{\prime}}>0.27\left(\frac{g_{Z^{\prime} q \bar{q}}}{g_{Z q \bar{q}}}\right)\left(\frac{g_{Z^{\prime} W W}}{g_{Z^{\prime} W W \max }}\right)\left(\frac{M_{Z^{\prime}}}{M_{Z}}\right)^{2} \\
\Gamma_{W^{\prime}}>0.40\left(\frac{g_{W^{\prime} q \bar{q}}}{g_{W q \bar{q}}}\right)\left(\frac{g_{W^{\prime} W Z}}{g_{W^{\prime} W Z_{\max }}}\right)\left(\frac{M_{W^{\prime}}}{M_{W}}\right)^{2} .
\end{gathered}
$$

We perform our parton-level analyses using the stand alone package of MADGRAPH [8] supplemented by the new states and interactions. We simulate experimental resolutions by smearing the energies, but not directions, of all final state leptons with a Gaussian error given by a resolution $\Delta E / E=0.1 / \sqrt{E} \oplus 0.01$ while for jets we assumed a resolution $\Delta E / E=0.5 / \sqrt{E} \oplus 0.03$, if $\left|\eta_{j}\right| \leq 3$, and $\Delta E / E=1 / \sqrt{E} \oplus 0.07$, if $\left|\eta_{j}\right|>3$ ( $E$ in $\mathrm{GeV}$ ). Furthermore the lepton detection efficiency was taken to be 0.9. In our calculations we use CTEQ6L parton distribution functions [9] with renormalization and factorization scales $\mu_{F}^{0}=\mu_{R}^{0}=\sqrt{\left(p_{T}^{\ell^{+2}}+p_{T}^{\ell^{-2}}\right) / 2}$ for pure leptonic final states, while for the channels containing jets we employ 
$\mu_{F}^{0}=\mu_{R}^{0}=\sqrt{\left(p_{T}^{j_{2}^{2}}+p_{T}^{j_{1}^{2}}\right) / 2}$.

\section{SIGNAL AND BACKGROUNDS}

In this section we describe the main features of the $Z^{\prime}$ and $W^{\prime}$ signals for the different final states, presenting the main SM backgrounds and discuss possible cuts to enhance the signal to backgrounds ratios.

$$
\text { A. } p \boldsymbol{p} \rightarrow Z^{\prime} \rightarrow W^{+} \boldsymbol{W}^{-} \rightarrow \boldsymbol{\ell}^{+} \boldsymbol{\ell}^{\prime-} \boldsymbol{E}_{\boldsymbol{T}}
$$

We start discussing the observability of the $Z^{\prime}$ signal in the leptonic $W^{ \pm}$decay channels with both equal and different flavor leptons in the final state. In the case of different flavor leptons, the $Z^{\prime}$ signal in process (1) possesses SM backgrounds coming from the production of $W^{+} W^{-}$pairs, as well as, from $t \bar{t}$ pairs where the top quarks decay semileptonically.

The starting cuts are meant to ensure the detection and isolation of the final partons plus a minimum transverse momentum since $Z^{\prime}$ has a mass in excess of a few hundred $\mathrm{GeV}[10,11]$ and it is expected to lead to hard leptons

$$
\left|\eta_{\ell}\right|<2.5, \quad \Delta R_{\ell \ell}>0.4 \text { and } p_{T}^{\ell}>50 \mathrm{GeV} \text {. }
$$

The presence of two neutrinos in the final state renders impossible the complete reconstruction of the event. Nevertheless the signal can still be partially characterized with the use of the transverse invariant mass variable

$$
\begin{aligned}
M_{T}^{W W}= & {\left[\left(\sqrt{\left(p_{T}^{\ell^{+} \ell^{\prime-}}\right)^{2}+m_{\ell^{+} \ell^{\prime-}}^{2}}+\sqrt{p_{T}^{2}+m_{\ell^{+} \ell^{\prime-}}^{2}}\right)^{2}\right.} \\
& \left.-\left(\vec{p}_{T}^{\ell^{+} \ell^{\prime-}}+\vec{p}_{T}\right)^{2}\right]^{1 / 2}
\end{aligned}
$$

where $\overrightarrow{\not p}_{T}$ is the missing transverse momentum vector, $\vec{p}_{T}^{\ell^{+} \ell^{\prime-}}$ is the transverse momentum of the pair $\ell^{+} \ell^{\prime-}$ and $m_{\ell^{+} \ell^{\prime-}}$ is the $\ell^{+} \ell^{\prime-}$ invariant mass.

We show in Fig. 1 the characteristic invariant mass distribution expected for several values of $M_{Z^{\prime}}$. The SM prediction is represented by the solid light grey (yellow) histogram while the darker areas represent the $Z^{\prime}$ expected signal for $M_{Z^{\prime}}=0.5$ and $1 \mathrm{TeV}$ as indicated. As seen in this figure, the characteristic peak associated with the production of a resonance is substantially broadened due to the incomplete event reconstruction, however, the $Z^{\prime}$ production is still signaled by an excess of events in the region

$$
M_{T}^{W W}>\frac{M_{Z^{\prime}}}{2} .
$$

A sizable contribution to the background arises from the SM $t \bar{t}$ production which leads to a $W^{+} W^{-}$pair on the final state accompanied by two $b$ jets. This background can be efficiently reduced by vetoing the presence of additional jets with

$$
\left|\eta_{j}\right|<3 \text { and } p_{T}^{j}>20 \mathrm{GeV}
$$

The probability of a QCD (electroweak) event to survive

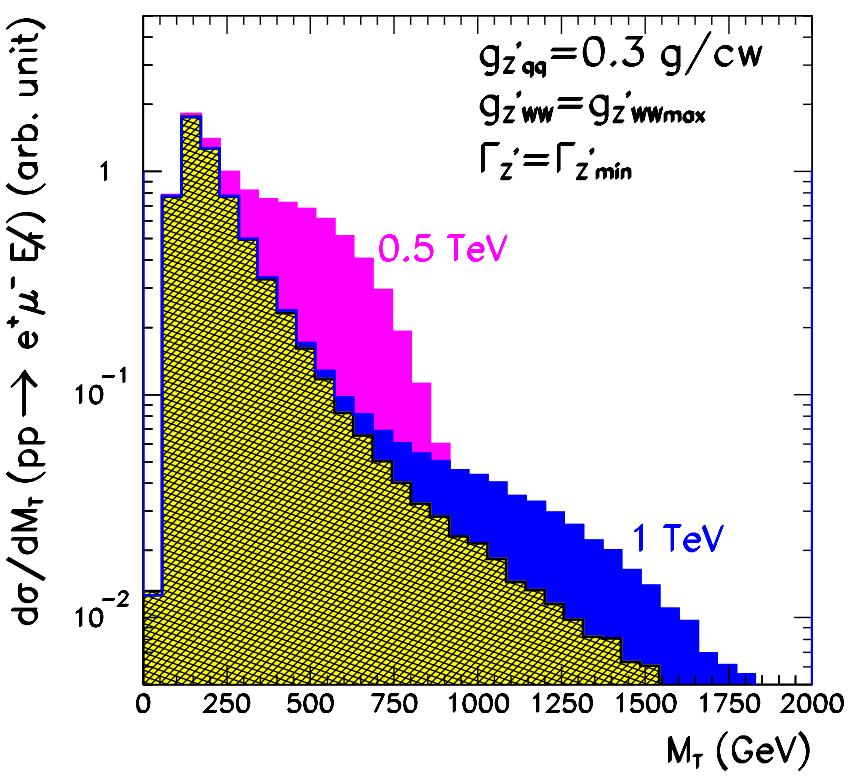

FIG. 1 (color online). Transverse mass distribution for the $p p \rightarrow e^{+} \mu^{-} \mathscr{E}_{T}$ final state for different values of $M_{Z^{\prime}}$. The widths are chosen to be the minimum values compatible with Eq. (6), $\Gamma_{\min }=4.7,37 \mathrm{GeV}$ for 0.5 and $1 \mathrm{TeV}$, respectively. The hatched area stands for the SM background contribution.

such a central jet veto has been analyzed for various processes in Ref. [12]. Moreover, at the high-luminosity run of the LHC there will be more than one interaction per bunch crossing, consequently there is a probability of detecting an extra jet in the gap region due to pile-up. In Ref. [13] it was estimated that survival probability due to pile-up is 0.75 for a threshold cut of $p_{T}=20 \mathrm{GeV}$. Taking into account these two effects we included in our analysis veto survival probabilities

$$
P_{\text {surv }}^{\mathrm{EW}}=0.56, \quad P_{\text {surv }}^{\mathrm{QCD}}=0.23 .
$$

Further reduction of $t \bar{t}$ background can be achieved by use of the difference of the azimuthal angular distributions of the final leptons. This behavior is illustrated in Fig. 2 where we show the azimuthal angular separation of the charged leptons for both signal and $t \bar{t}$ background. Consequently, one can also require

$$
\Delta \phi_{\ell \ell^{\prime}}<2.5 \text {. }
$$

In the case that the final state leptons in reaction (1) have the same flavor $(e e$ or $\mu \mu)$, there are additional backgrounds originating from Drell-Yan lepton pair production, as well as $Z Z$ pair production. In this case, the cuts (10), (12), (13), and (15) are supplemented by

$$
\not_{T}>50 \mathrm{GeV}
$$

in order to suppress the Drell-Yan pair production. Furthermore 


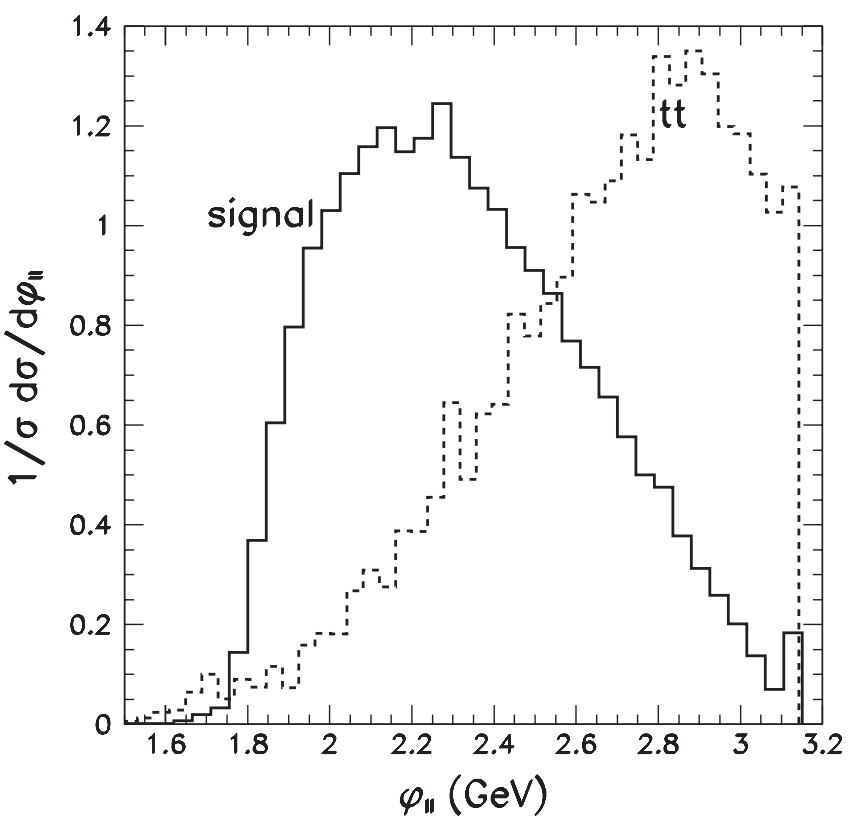

FIG. 2. Distribution of the azimuthal angular separation of the charged leptons for the $\ell^{+} \ell^{\prime-} \mathscr{E}_{T}$ final state. The solid histogram stands for the signal distribution for $M_{Z^{\prime}}=0.5 \mathrm{TeV}$ with $\Gamma_{Z^{\prime}} / M_{Z^{\prime}}=0.1$, while the dashed one represents the SM $t \bar{t}$ background.

$$
m_{\ell^{+} \ell^{-}}>100 \mathrm{GeV}
$$

is required to reduce the $Z Z$ background.

Table I contains the cross sections for the different contributions to the SM backgrounds after all the cuts described above, as well as for the signal, normalized as in Eq. (5), for $M_{Z^{\prime}}=0.5,1$, and $1.5 \mathrm{TeV}$ and for an illustrative value of the width $\Gamma_{Z^{\prime}}=0.05 M_{Z^{\prime}}$. Once the cuts are imposed, the interference term $\sigma_{\text {int }}$ is negligibly small for all values of $Z^{\prime}$ masses and widths considered.

\section{B. $p p \rightarrow Z^{\prime} \rightarrow W^{+} W^{-} \rightarrow \ell^{ \pm} j j \mathbb{E}_{T}$}

A sharper $Z^{\prime}$ signal can be obtained by the study of the channel where one $W$ decays leptonically while the other decays hadronically. This final state can be reconstructed,

TABLE I. Signal and background cross sections in fb for the $\ell^{+} \ell^{\prime-} \mathscr{E}_{T}$ final state for all possible lepton combinations with either electrons or muons. For the final state with different flavor leptons the cuts (10) to (15) are applied while for same flavor leptons the cuts (10) to (17) are imposed. These results do not include the lepton detection efficiencies nor the gap survival probabilities. For the signal the results are show for an illustrative value of the width $\Gamma_{Z^{\prime}}=0.05 M_{Z^{\prime}}$.

\begin{tabular}{lccc}
\hline \hline$M_{Z^{\prime}}(\mathrm{TeV})$ & $\sigma_{\mathrm{SM}}^{E W}(\mathrm{fb})$ & $\sigma_{\mathrm{SM}}^{t \bar{t}}(\mathrm{fb})$ & $\sigma_{Z^{\prime}}(\mathrm{fb})$ \\
\hline 0.5 & 184 & 10.8 & 793 \\
1.0 & 92.5 & 1.88 & 361 \\
1.5 & 52 & 0 & 158 \\
\hline \hline
\end{tabular}

up to a twofold ambiguity on the neutrino longitudinal momentum, exhibiting the characteristic peak of a resonance production. On the other hand this channel possesses large QCD backgrounds due to the $W j j$ production, as well as, $Z j j$ with the $Z$ decaying leptonically and one of the leptons being missed. $t \bar{t}$ production also contribute to the background, however, presenting additional $b$ jets.

Again the starting cuts are meant to ensure the detection and isolation of the final partons:

$$
\left|\eta_{\ell}\right|<2.5, \quad\left|\eta_{j}\right|<3 \quad \text { and } \quad \Delta R_{j j(j \ell)}>0.4 \text {. }
$$

The large QCD background can be reduced by making use of the characteristic harder transverse momenta of charged lepton and the jets in the $Z^{\prime}$ signal. As illustration we show in Fig. 3 the $p_{T}^{\ell}$ distribution for both the sum of the backgrounds as well as for the signal for several values of $M_{Z^{\prime}}$. Thus, the signal to background ratio can be improved by requiring the lepton and jets to be energetic. We find the following suitable set of variables:

$$
p_{T}^{\ell}>p_{T}^{\ell \min }, \quad p_{T}^{j}>\left.p_{T}^{j \min } \quad p_{T}^{j}\right|_{\max }>p_{T}^{\max }, \quad \not \mathbb{E}_{T}>\mathbb{E}_{T}^{\min },
$$

where we denoted the jet largest transverse momentum as $\left.p_{T}^{j}\right|_{\max }$. The optimum cuts depend on the $Z^{\prime}$ mass as given in Table II.

The identification of the hadronically decaying $W$ is accomplished requiring that the dijet invariant mass is compatible with the $W$ mass, i.e.

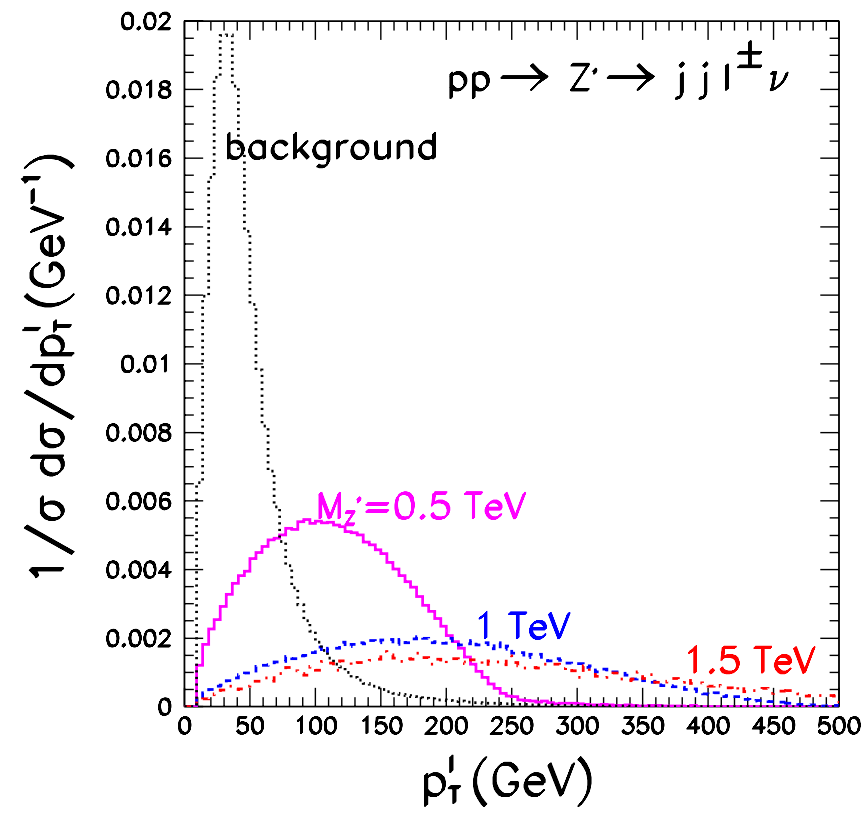

FIG. 3 (color online). Transverse momentum distribution for the charge lepton in the $\ell^{ \pm} j j \mathscr{E}_{T}$ final state. The dotted histogram corresponds to the expected distribution for the background after the cuts in Eq. (18) while the solid, dashed, and dot-dashed line correspond to the expected distribution for the signal with $M_{Z^{\prime}}=$ $0.5,1$ and $1.5 \mathrm{TeV}$, respectively, and for $\Gamma_{Z^{\prime}}=0.05 M_{Z^{\prime}}$. 
TABLE II. Cuts for $p p \rightarrow Z^{\prime} \rightarrow W^{+} W^{-} \rightarrow \ell^{ \pm} j j \mathscr{E}_{T}$ as a function of the new resonance mass.

\begin{tabular}{lrrr}
\hline \hline$M_{Z^{\prime}}(\mathrm{TeV})$ & 0.5 & 1.0 & 1.5 \\
\hline$p_{T}^{\ell \min }(\mathrm{GeV})$ & 75 & 100 & 100 \\
$p_{T}^{j \min }(\mathrm{GeV})$ & 60 & 75 & 100 \\
$p_{T}^{\max }(\mathrm{GeV})$ & 110 & 75 & 100 \\
$\mathscr{E}_{T}^{\min }(\mathrm{GeV})$ & 50 & 75 & 75 \\
$\delta(\mathrm{GeV})$ & 50 & 100 & 200 \\
\hline \hline
\end{tabular}

$$
\left|M_{j j}-M_{W}\right|<10 \mathrm{GeV} .
$$

As discussed in Sec. III A, the $t \bar{t}$ background can be further reduced by vetoing additional jets in the central region through the requirement (13) which we assumed to lead to the corresponding veto survival probabilities (14). This veto also suppresses the QCD processes since QCD events presents a higher probability of emitting additional jets [12].

As mentioned above, the presence of just one neutrino in the final state permits the reconstruction of its momentum by imposing the transverse momentum conservation and requiring that the invariant mass of the neutrino- $\ell^{ \pm}$pair is $M_{W}$; this procedure leads a twofold ambiguity on neutrino longitudinal momentum. This opens up the possibility of reconstructing the $Z^{\prime}$ Breit-Wigner profile to better pinpoint this state. Using the two possibilities for the neutrino momentum we evaluated the invariant mass of the $\ell^{ \pm} j j \ddot{E}_{T}$ final state. In Fig. 4 we show the reconstructed invariant mass distribution using the minimum of the two $W W$ reconstructed masses, $M_{\mathrm{rec}}^{\min }$, after the cuts in Eqs. (18)(20).

For any of the widths considered in the analysis, the $Z^{\prime}$ peak can be efficiently observed by requiring:

$$
\left|M_{\mathrm{rec}}^{\min }-M_{Z^{\prime}}\right|<\delta,
$$

where $\delta$ is chosen to guarantee that for a given mass and for the range of widths considered most of the signal is within this invariant mass window. The chosen values of $\delta$ are given in Table II.

We present in Table III the signal and background cross sections for the $\ell^{ \pm} j j \mathscr{E}_{T}$ channel after applying the cuts (18) to (21). ${ }^{1}$ From this table, we can see that the cross sections are drastically reduced for $Z^{\prime}$ masses in excess of $\simeq 1 \mathrm{TeV}$. In this region of the parameter space the $W$ 's are very energetic, thus, their decay products are highly collimated not passing the isolation cuts in (18). This is illustrated in Fig. 5 where we show the $\Delta R_{j j}$ distribution expected from the $Z^{\prime}$ signal for different values of $M_{Z^{\prime}}$ when only the rapidity cuts in Eq. (18) are imposed. As seen in the figure, already for $M_{Z^{\prime}}=1.5 \mathrm{TeV}$ a sizeable

\footnotetext{
${ }^{1}$ Again, once the cuts are imposed, the interference term $\sigma_{\text {int }}$ is negligibly small for all values of $Z^{\prime}$ masses and widths considered.
}

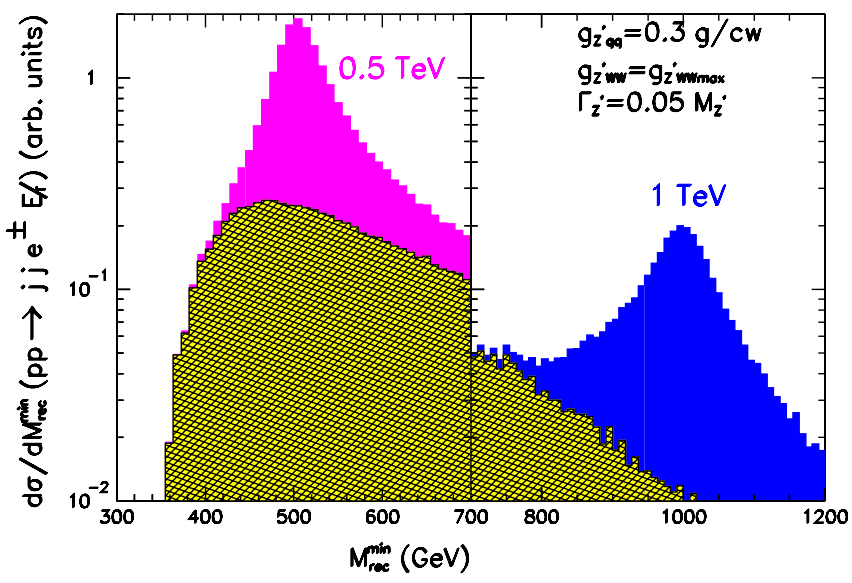

FIG. 4 (color online). In the left (right) panel we present the reconstructed minimum invariant mass distribution for the $p p \rightarrow$ $j j \ell^{ \pm} \mathscr{E}_{T}$ final state for $M_{Z^{\prime}}=0.5 \mathrm{TeV}(1 \mathrm{TeV})$ over the corresponding SM backgrounds (hatched area) after the cuts in Eqs. (18)-(20). Despite the arbitrary overall normalization the figure reflects the relative size of the two resonance signals (and of their corresponding backgrounds).

fraction of the signal leads to very collimated jets, which results into the signal reduction once the isolation cuts are imposed.

In order to overcome this suppression one can consider the possibility of the final state $\ell^{ \pm} j \mathscr{E}_{T}$, as discussed in Ref. [14]. The realistic evaluation of the attainable level of rejection of the QCD background for this case requires a devoted study including details of the detector simulation, which is beyond the scope of this work. We therefore keep the isolation cut which may render our conclusions of observability of the heavy resonances conservative.

$$
\text { C. } p \boldsymbol{p} \rightarrow W^{\prime} \rightarrow W^{ \pm} Z \rightarrow \ell^{\prime \pm} \ell^{+} \ell^{-} \boldsymbol{E}_{T}
$$

In this channel, the $W^{\prime}$ production is characterized by the presence of three charged leptons $\left(e^{ \pm}\right.$or $\left.\mu^{ \pm}\right)$and missing transverse energy. The primary SM background to this signal is the $W^{ \pm} Z$ and $Z Z$ productions, where one of the final state leptons evades detection in the $Z Z$ case. The $t \bar{t}$ pair production also contributes to the SM background, however, since it requires that one of the isolated leptons

TABLE III. Signal and background cross sections in fb for the final state $\ell^{ \pm} j j \mathscr{E}_{T}$ after imposing the cuts (18) to (21) summed over $\ell^{ \pm}$being an electron or a muon. These results do not include the jet and lepton reconstruction efficiencies. For the signal the results are show for an illustrative value of the width $\Gamma_{Z^{\prime}}=0.05 M_{Z^{\prime}}$.

\begin{tabular}{lcccc}
\hline \hline$M_{Z^{\prime}}(\mathrm{TeV})$ & $\sigma_{\mathrm{SM}}^{E W}(\mathrm{fb})$ & $\sigma_{\mathrm{SM}}^{\mathrm{QCD}}(\mathrm{fb})$ & $\sigma_{\mathrm{SM}}^{t \bar{t}}(\mathrm{fb})$ & $\sigma_{Z^{\prime}}(\mathrm{fb})$ \\
\hline 0.5 & 17.6 & 208 & 19.2 & 1232 \\
1.0 & 5.6 & 46 & 5.6 & 270 \\
1.5 & 1.5 & 12 & 1.8 & 9.5 \\
\hline \hline
\end{tabular}




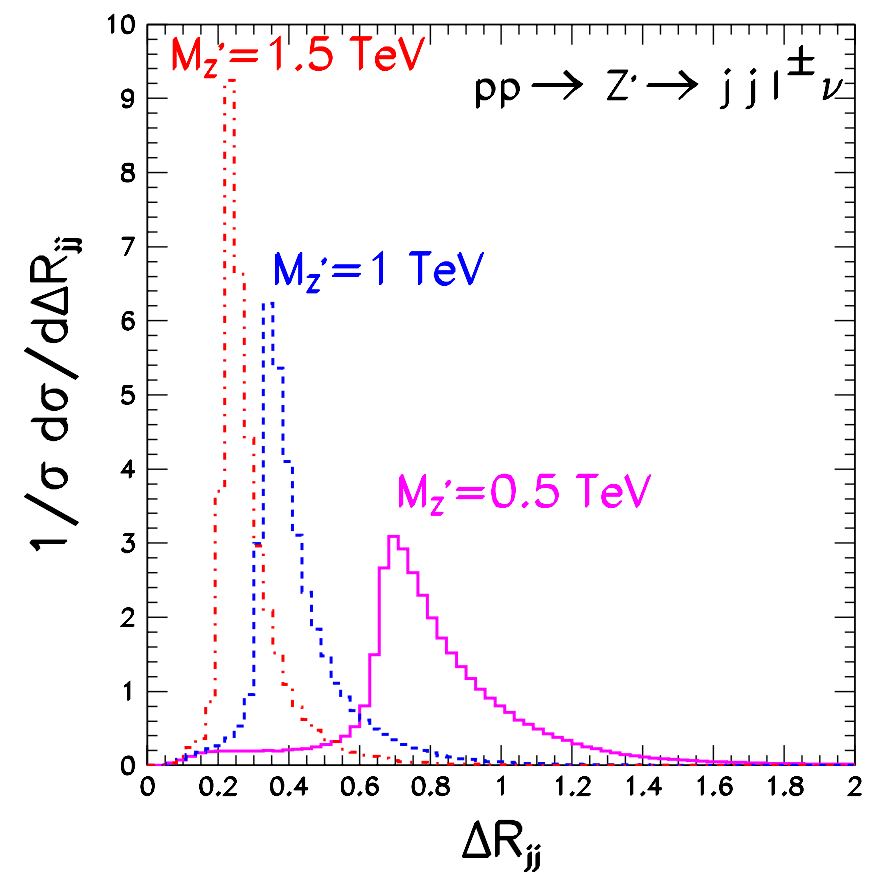

FIG. 5 (color online). $\Delta R_{j j}$ distribution in the $\ell^{ \pm} j j \mathbb{E}_{T}$ final state for different $M_{Z^{\prime}}$ signals as labeled in the figures. In all cases $\Gamma_{Z^{\prime}}=0.05 M_{Z^{\prime}}$.

originates from a $b$ quark semileptonic decay it is very suppressed.

We start with the minimum cuts to ensure the detection and isolation of the final leptons:

$$
\left|\eta_{\ell}\right|<2.5, \quad \Delta R_{\ell \ell}>0.4 \text { and } p_{T}^{\ell}>10 \mathrm{GeV} .
$$

In the search for a lighter resonance, i.e. $M_{W^{\prime}}=0.5 \mathrm{TeV}$, a further cut is needed to tame the SM backgrounds, demanding that the hardest observed lepton has transverse momentum in excess of $120 \mathrm{GeV}$.

In the signal the final state contains a pair of same flavor opposite charge (SFOC) leptons compatible with the production of a $Z$. Thus the signal gets enhanced by requiring

$$
\left|M_{\ell \ell}^{\mathrm{SFOC}}-M_{Z}\right|<20 \mathrm{GeV} \text {. }
$$

As in Sec. III B, the presence of just one final state neutrino in process (2) allows for the reconstruction of the neutrino momentum. Consequently it is possible to evaluate the total $\ell \ell \ell^{\prime} \nu$ invariant mass up to a twofold ambiguity. Thus one can enhance the signal over SM backgrounds by requiring that the total reconstructed invariant mass satisfies

$$
\left|M_{\mathrm{rec}}^{\min }-M_{W^{\prime}}\right|<\delta,
$$

where $M_{\mathrm{rec}}^{\min }$ is the smaller of the two solutions for the reconstructed invariant mass. We chose the size of the window around $M_{W^{\prime}}$ as given in Table II with the implicit understanding that $M_{Z^{\prime}}$ should be taken as the $W^{\prime}$ mass.
TABLE IV. Signal and background cross sections for the $\ell^{\prime \pm} \ell^{+} \ell^{-} \mathscr{E}_{T}$ final state after imposing cuts (22)-(24). Contributions from all trilepton combinations with electrons and muons are included. Backgrounds include both irreducible and reducible contributions plus the top backgrounds. The lepton detection efficiencies are not included. The signal cross section is given for $\Gamma_{W^{\prime}}=0.05 M_{W^{\prime}}$.

\begin{tabular}{lcc}
\hline \hline$M_{W^{\prime}}(\mathrm{TeV})$ & $\sigma_{\mathrm{SM}}(\mathrm{fb})$ & $\sigma_{W^{\prime}}(\mathrm{fb})$ \\
\hline 0.5 & 0.87 & 297 \\
1.0 & 0.14 & 85 \\
1.5 & 0.01 & 6.4 \\
\hline \hline
\end{tabular}

We present in Table IV the signal and SM cross sections after cuts (22)-(24). The interference term $\sigma_{\text {int }}$ is negligibly small for all values of $W^{\prime}$ masses and widths considered. As we can see, this channel presents a small SM background due to the reduced leptonic branching ratio of the $W Z$ final state. However, the signal cross section is depleted as well. As discussed in the previous subsection, the collimation of the $Z$ decay products reduces the signal for large $M_{W^{\prime}}$ due to the requirement of the lepton isolation cuts.

$$
\text { D. } \boldsymbol{p} \boldsymbol{p} \rightarrow \boldsymbol{W}^{\prime} \rightarrow \boldsymbol{W}^{ \pm} \boldsymbol{Z} \rightarrow \boldsymbol{\ell}^{ \pm} \boldsymbol{j} \boldsymbol{j} \boldsymbol{E}_{\boldsymbol{T}}
$$

The final state $\ell^{ \pm} j j \mathbb{E}_{T}$ can also be used to study $W^{\prime}$ production, in addition to $Z^{\prime}$ searches described in Sec. III B. Since the $W$ and $Z$ masses are relatively close, many events might be classified as $Z^{\prime}$ and $W^{\prime}$ productions if the new vector resonances possess similar masses. In our analyses we assumed that these new states are not degenerated so we did not add their contributions to this final state.

The $W^{\prime}$ and $Z^{\prime}$ signals are similar for this final state, therefore, the same cuts (18) to (21) can be applied with the obvious change of $M_{W}$ to $M_{Z}$ in (20) and of $M_{Z^{\prime}}$ by $M_{W^{\prime}}$ in (21). Also to efficiently suppress the $t \bar{t}$ and QCD backgrounds the vetoing of additional jets in the central region (13) is imposed with the corresponding veto survival probabilities (14).

Table $\mathrm{V}$ shows the signal and background cross sections for the $W^{\prime}$ search in the $\ell^{ \pm} j j \not E_{T}$ channel. Comparison with

TABLE V. $W^{\prime}$ signal and SM background cross sections for the final state $\ell^{ \pm} j j \mathscr{E}_{T}$ as a function of the $W^{\prime}$ mass. Results are summed over $\ell^{ \pm}$being an electron or a muon and do not include the jet and lepton reconstruction efficiencies. For the signal the results are show for an illustrative value of the width $\Gamma_{W^{\prime}}=$ $0.05 M_{W^{\prime}}$.

\begin{tabular}{lcccc}
\hline \hline$M_{W^{\prime}}(\mathrm{TeV})$ & $\sigma_{\mathrm{SM}}^{E W}(\mathrm{fb})$ & $\sigma_{\mathrm{SM}}^{\mathrm{QCD}}(\mathrm{fb})$ & $\sigma_{\mathrm{SM}}^{t \bar{t}}(\mathrm{fb})$ & $\sigma_{W^{\prime}}(\mathrm{fb})$ \\
\hline 0.5 & 13.2 & 210 & 11.4 & 1354 \\
1.0 & 4.0 & 50 & 4.4 & 673 \\
1.5 & 1.5 & 14 & 0.88 & 32 \\
\hline \hline
\end{tabular}


Table IV shows that in this channel the signal has a higher statistics than the purely leptonic mode due to the larger $Z$ hadronic branching ratio. However, as seen in the table, even after all the cuts imposed, this channel still suffers a large QCD background.

$$
\text { E. } p \boldsymbol{p} \rightarrow \boldsymbol{W}^{\prime} \rightarrow \boldsymbol{W}^{ \pm} \boldsymbol{Z} \rightarrow \boldsymbol{\ell}^{+} \ell^{-} \boldsymbol{j} \boldsymbol{j}
$$

Finally we study the final state $\ell^{+} \ell^{-} j j$ for which the full reconstruction of the $W^{\prime}$ signal as well as the $Z$ and $W$ intermediate states is possible. This, as shown below, allows for a very efficient reduction of the SM backgrounds.

The centrality, isolation and momentum cuts for the final partons for this channel are:

$$
\begin{array}{rll}
\left|\eta_{\ell}\right|<2.5, \quad\left|\eta_{j}\right|<3 \text { and } & \Delta R_{j j(j \ell)(\ell \ell)}>0.4 . \\
p_{T}^{\ell}>50, & (100), & {[100] \mathrm{GeV}} \\
p_{T}^{j}>70, & (100), & {[100] \mathrm{GeV}}
\end{array}
$$

for $M_{W^{\prime}}=0.5,(1.0)$, [1.5] TeV.

The identification of the hadronically decaying $W$ and leptonically decaying $Z$ is obtained by requiring:

$$
\left|M_{j j}-M_{W}\right|<10 \mathrm{GeV}, \quad\left|M_{\ell^{+} \ell^{-}}-M_{Z}\right|<10 \mathrm{GeV} .
$$

In Fig. 6 we show the reconstructed invariant mass distribution $M_{j j \ell^{+} \ell^{-}}$after the cuts in Eqs. (25)-(27). As seen in this figure, in this channel the $W^{\prime}$ invariant mass can be well reconstructed. We consequently compute the signal cross section for $M_{W^{\prime}}=0.5$ (1) [1.5] TeV by requiring

$$
\left|M_{j j \ell^{+} \ell^{-}}-M_{W^{\prime}}\right|<50, \quad(100), \quad[200] \mathrm{GeV} \text {. }
$$

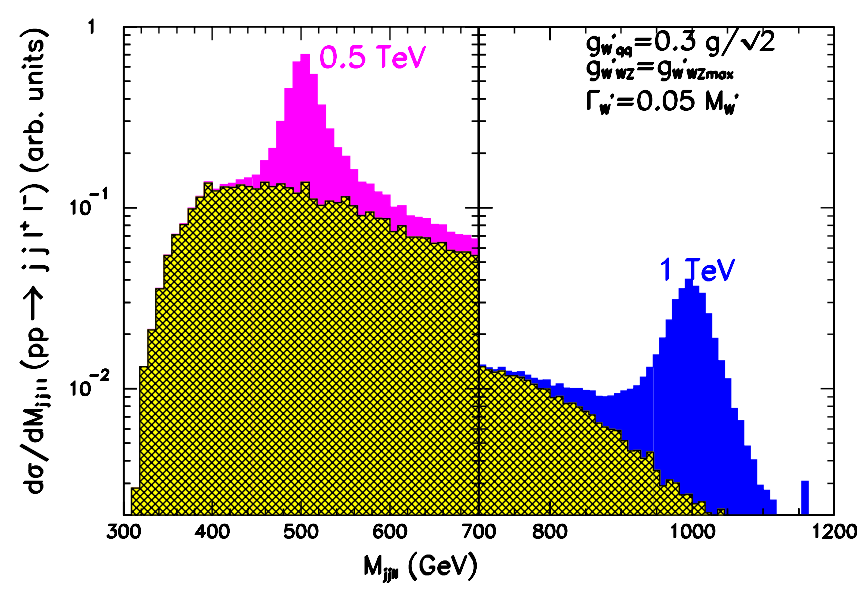

FIG. 6 (color online). The left (right) panel contains the reconstructed invariant mass distribution for the $p p \rightarrow j j \ell^{+} \ell^{-}$ final state and for $M_{Z^{\prime}}=0.5$ (1) $\mathrm{TeV}$ over their corresponding backgrounds, depicted as a hatched area, after the cuts given by Eqs. (25)-(27). The relative size of the signals and backgrounds between the two resonances is maintained despite the arbitrary overall normalization.
TABLE VI. $W^{\prime}$ signal and SM background cross sections for the final state $\ell^{+} \ell^{-} j j$ as a function of the $W^{\prime}$ mass. Results are summed over $\ell^{ \pm}$being an electron or a muon and do not include the jet and lepton reconstruction efficiencies. For the signal the results are show for an illustrative value of the width $\Gamma_{W^{\prime}}=$ $0.05 M_{W^{\prime}}$.

\begin{tabular}{lccc}
\hline \hline$M_{W^{\prime}}(\mathrm{TeV})$ & $\sigma_{\mathrm{SM}}^{E W}(\mathrm{fb})$ & $\sigma_{\mathrm{SM}}^{\mathrm{QCD}}(\mathrm{fb})$ & $\sigma_{W^{\prime}}(\mathrm{fb})$ \\
\hline 0.5 & 12.2 & 17.2 & 380 \\
1.0 & 0.14 & 0.76 & 51 \\
1.5 & 0.03 & 0.12 & 3.3 \\
\hline \hline
\end{tabular}

We give in Table VI the signal and SM cross sections after cuts (25)-(28). The $t \bar{t}$ cross section is reduced to a negligible size after these cuts are imposed. The interference term $\sigma_{\text {int }}$ is negligibly small for all values of $W^{\prime}$ masses and widths considered.

As in the previous section, the collimation of the $Z$ and $W$ decay products reduces the signal for large $M_{W^{\prime}}$ due to the requirement of the lepton and jet isolation cuts imposed to ensure efficient reconstruction of the $Z$ and $W$ invariant masses.

\section{RESULTS AND CONCLUSIONS}

In the present study, we use as signal of the production of a new resonance $V^{\prime}\left(=Z^{\prime}\right.$ or $\left.W^{\prime}\right)$ the excess in the total number of events over the SM expectations after the application of the suitable cuts described in the previous section.

The analysis of the statistical significance of the signals described is simplified by the fact that in all channels the interference between the SM and the $V^{\prime}$ contributions, $\sigma_{\text {int }}\left(M_{V^{\prime}}, \Gamma_{V^{\prime}}\right)$, is negligible for $\Gamma_{V^{\prime}}<M_{V^{\prime}} / 2$ after cuts. In particular, for sufficiently high number of expected background events, the $5 \sigma$ sensitivity bounds on the $V^{\prime}$ properties can be obtained assuming Gaussian statistics as

$$
\left(\frac{g_{V^{\prime} q \bar{q}}}{g_{V q \bar{q}}} \frac{g_{V^{\prime} W V}}{g_{V^{\prime} W V \max }}\right)^{2}>5 \frac{\sqrt{\sigma_{\mathrm{SM}}}}{\sqrt{\mathcal{L}} \sigma_{V^{\prime}}\left(M_{V^{\prime}}, \Gamma_{V^{\prime}}\right)} .
$$

Here we present our results for an integrated luminosity of $100 \mathrm{fb}^{-1}$. For this luminosity the number of background events is large enough to Gaussian statistics to hold for all cases with the exception of the leptonic heavy $W^{\prime}$ channel, $p p \rightarrow W^{\prime} \rightarrow W^{ \pm} Z \rightarrow \ell^{\prime \pm} \ell^{+} \ell^{-} \mathscr{E}_{T}$. In this case condition (29) is modified by adopting the corresponding $5 \sigma$ observability bound for Poisson statistics in the presence of the corresponding expected background.

We depict in Figs. 7-11 the ranges of couplings $g_{V^{\prime} q \bar{q}} g_{V^{\prime} W V}$ for which a $5 \sigma$ signal can be observed as a function of the resonance width for three different values of its mass $M_{V^{\prime}}=0.5,1,1.5 \mathrm{TeV}$ in the different channels. There are some basic features that are common to all the cases. First of all, the observability regions are bounded both from below and above. The lower bound arises from 


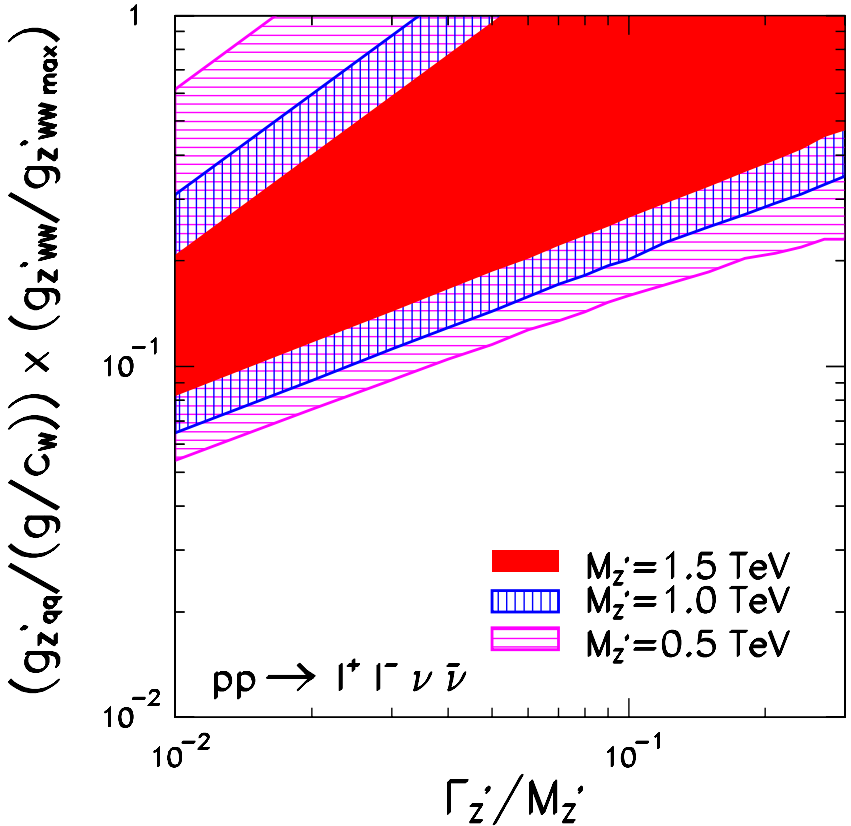

FIG. 7 (color online). The filled regions are the ranges of the parameters for observation of a $Z^{\prime}$ with mass $M_{Z^{\prime}}=0.5,1$, and $1.5 \mathrm{TeV}$ with at least $5 \sigma$ significance in the reaction $p p \rightarrow Z^{\prime} \rightarrow$ $W^{+} W^{-} \rightarrow \ell^{+} \ell^{-} \mathbb{E}_{T}$.

the condition of the signal being observable while the upper limit originates from the consistency of our effective description of the vector resonance production. For a given vector mass the contribution of the $V^{\prime} \rightarrow V V$ and $V^{\prime} \rightarrow q \bar{q}^{\prime}$ partial widths should not surpass the assumed value of the

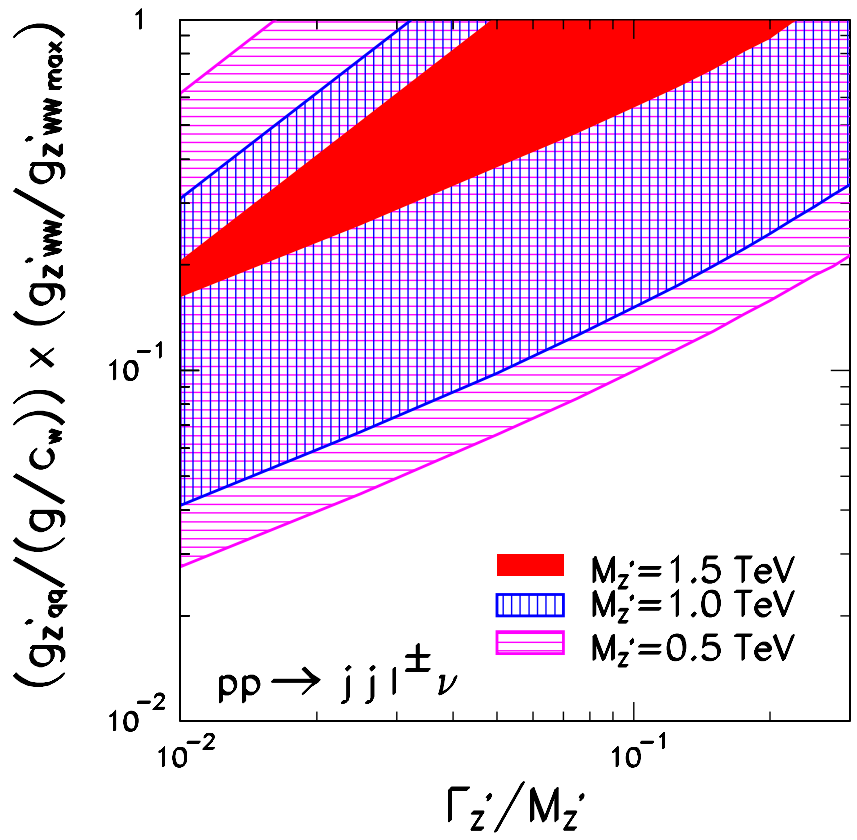

FIG. 8 (color online). Same as Fig. 7 for the process $p p \rightarrow$ $Z^{\prime} \rightarrow W^{+} W^{-} \rightarrow \ell^{ \pm} j j \ddot{E}_{T}$.

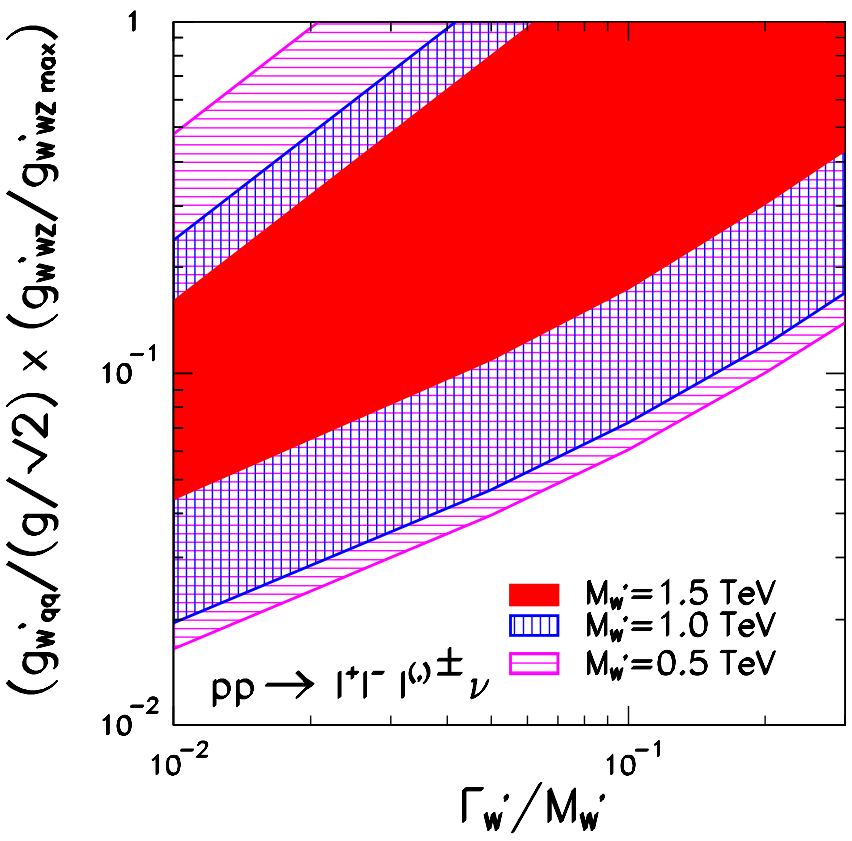

FIG. 9 (color online). The filled regions are the ranges of the parameters for observation of a $W^{\prime}$ with mass $M_{W^{\prime}}=0.5$, 1 , and $1.5 \mathrm{TeV}$ with at least $5 \sigma$ significance in the channel $p p \rightarrow W^{\prime} \rightarrow$ $W^{ \pm} Z \rightarrow \ell^{+} \ell^{-} \ell^{ \pm} \mathscr{E}_{T}$.

total width; this fact is described by the upper limits given in Eqs. (8) and (9).

Another general feature is that the lower bound on the $g_{V^{\prime} q \bar{q}} g_{V^{\prime} W V}$ increases as the total width $\Gamma_{V^{\prime}}$ is augmented just because broad resonances tend to produce more events outside the $V^{\prime}$ invariant mass window used to define the

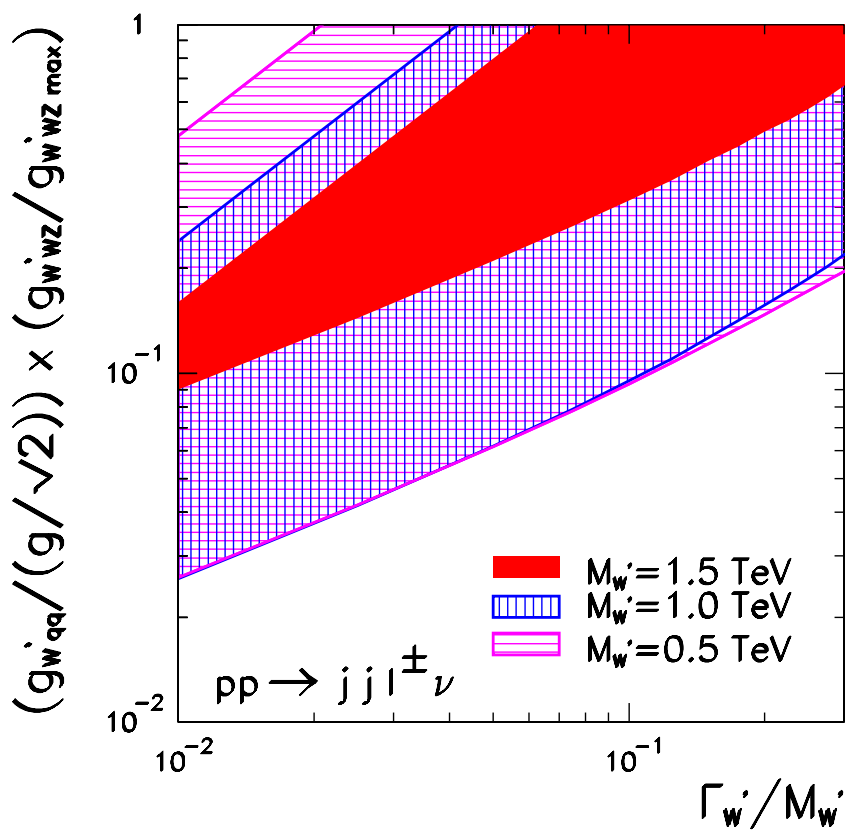

FIG. 10 (color online). Same as Fig. 9 for the reaction $p p \rightarrow$ $W^{\prime} \rightarrow W^{ \pm} Z \rightarrow \ell^{ \pm} j j \mathbb{E}_{T}$. 


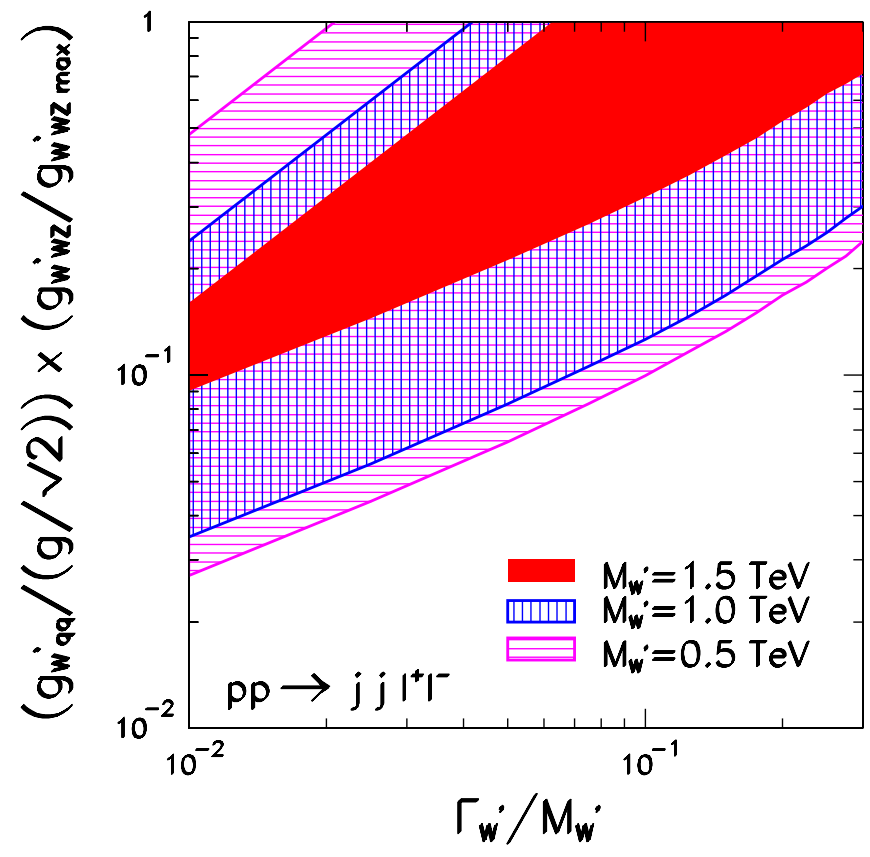

FIG. 11 (color online). Same as Fig. 9 for the process $p p \rightarrow$ $W^{\prime} \rightarrow W^{ \pm} Z \rightarrow \ell^{ \pm} \ell^{ \pm} j j$.

signal; see for instance (21). Moreover, as can be expected, the lower limit on the coupling constant product increases for heavier $V^{\prime}$ masses. Here, we have two effects collaborating: not only the production cross section decreases for heavier vector masses, but also its decay products are more collimated becoming harder to pass the isolation cuts imposed on jets and leptons.

It is interesting to compare the expected sensitivity for the $Z^{\prime}$ in the purely leptonic $\ell^{+} \ell^{-} \mathbb{E}_{T}$ final state, shown in Fig. 7, with the semileptonic final state, $\ell^{ \pm} j j \mathscr{E}_{T}$, presented in Fig. 8. From these figures, we see that the possibility of reconstructing the $Z^{\prime}$ peak and the larger event rates leads to better sensitivity for the semileptonic channel for $M_{Z^{\prime}}=$ 0.5 and $1 \mathrm{TeV}$ despite the larger QCD backgrounds. Nevertheless, the importance of the channels is reversed for heavier $M_{Z^{\prime}}$ because the $W^{\prime}$ 's are very energetic and their decay jets are highly collimated not passing the isolation cut in (18).

As for the expected sensitivity to the $W^{\prime}$, the best channel for all masses considered is the purely leptonic final state $\ell^{\prime \pm} \ell^{+} \ell^{-} \mathbb{E}_{T}$ as consequence of the achievable background reduction. Also, as foreseeable, the predicted sensitivity to $W^{\prime}$ in the $\ell^{ \pm} j j \mathbb{E}_{T}$ final state is comparable to the corresponding one for $Z^{\prime}$ in the same final state (up to the difference strength in the couplings to light quarks of the $W$ and $Z$ ) and only slightly better than the observability of $W^{\prime}$ in the $\ell^{+} \ell^{-} j j$ channel.

The purely leptonic and semileptonic channels are complementary since they allow us to determine which resonance $\left(W^{\prime}\right.$ or $Z^{\prime}$ ) has been produced if a signal is indeed observed. The $W^{\prime}$ and $Z^{\prime}$ productions lead to the same final state $\ell^{ \pm} j j \mathscr{E}_{T}$, however, additional signals in the $\ell^{+} \ell^{-} j j$ and $\ell^{\prime \pm} \ell^{+} \ell^{-} \mathbb{E}_{T}$ channels characterize the $W^{\prime}$ existence, while the smoking gun for the $Z^{\prime}$ is an additional signal in the $\ell^{+} \ell^{\prime-} \mathbb{E}_{T}$ channel.

We also notice that should the narrow width approximation hold for $\sigma_{V^{\prime}}$ one would have

$$
\sigma_{V^{\prime}} \propto \sigma\left(p p \rightarrow V^{\prime}\right) \times B r\left(V^{\prime} \rightarrow W V\right)=A \frac{\left(g_{V^{\prime} q \bar{q}} g_{V^{\prime} W V}\right)^{2}}{\Gamma_{V^{\prime}}}
$$

where $A$ depends only on $M_{V^{\prime}}$. Thus, in the region of validity of the narrow width approximation, the bounds on $V^{\prime}$ obey a simple scaling law characterized by

$$
\left(\frac{g_{V^{\prime} q \bar{q}}}{g_{V q \bar{q}}} \frac{g_{V^{\prime} W V}}{g_{V^{\prime} W V \max }}\right)^{2} \times \Gamma_{V^{\prime}}
$$

being a constant depending on $M_{V^{\prime}}$ and the final state considered. As we can see in Figs. 7 to 11 our results are reasonably well described by Eq. (31) for $\Gamma_{Z^{\prime}} \lesssim$ $0.05-0.1 M_{Z^{\prime}}$, while there is a departure from this simple scaling rule for larger widths.

As a final comment, let us notice that these results should be taken with a pitch of salt since there might exist additional backgrounds to the vector resonance searches coming from the new physics associated to the electroweak symmetry breaking sector. For instance, in extra dimension models there is a plethora of new heavy states that might give rise to final states similar to the ones we studied, e.g. KK charged leptons can decay into a $W$ pair accompanied by neutrinos. If these backgrounds turned out to be sizeable, certainly the reconstruction of the new resonance Breit-Wigner profile would become an important tool to isolate the new vector states. Moreover, we have not considered the systematic uncertainties associated to the prediction of the absolute value of the SM backgrounds that can be of the order of 5-20\% [15].

In summary, in this work we have performed a model independent analyses of the observability of a new spin-1 particle, either neutral or charged, that is associated to the unitarity restoration in the electroweak vector boson scattering. In addition to the essential couplings to electroweak gauge boson pairs we also considered the new vector resonance couplings to light quarks; many extensions of the SM, like Higgsless models, contain such couplings. We analyzed the five channels shown in Eqs. (1) and (2) expressing the attainable bounds as a function of the resonance effective couplings, its mass and width. Our analyses show that the study of these process have a large reach at the LHC, allowing to discover or exclude many scenarios like Higgsless models.

\section{ACKNOWLEDGMENTS}

M. C. G-G thanks the CERN Theory Group for their hospitality during the final stages of this work. This work 
was partially supported by Conselho Nacional de Desenvolvimento Científico e Tecnológico (CNPq) and by Fundação de Amparo à Pesquisa do Estado de São Paulo (FAPESP). M. C. G-G is supported by the National
Science Foundation under Grant PHY-0354776 and by Spanish Grants FPA-2007-66665-C02-01 and CSD20080037.
[1] B. W. Lee, C. Quigg, and H. B. Thacker, Phys. Rev. D 16, 1519 (1977).

[2] S. Dimopoulos and L. Susskind, Nucl. Phys. B155, 237 (1979); L. Susskind, Phys. Rev. D 20, 2619 (1979); S. Weinberg, Phys. Rev. D 19, 1277 (1979).

[3] See for instance, C. T. Hill and E. H. Simmons, Phys. Rep. 381, 235 (2003); 390, 553(E) (2004).

[4] C. Csaki, C. Grojean, H. Murayama, L. Pilo, and J. Terning, Phys. Rev. D 69, 055006 (2004).

[5] C. Csaki, C. Grojean, H. Murayama, L. Pilo, and J. Terning, Phys. Rev. D 69, 055006 (2004); C. Csaki, C. Grojean, L. Pilo, and J. Terning, Phys. Rev. Lett. 92, 101802 (2004); Y. Nomura, J. High Energy Phys. 11 (2003) 050; C. Csaki, C. Grojean, J. Hubisz, Y. Shirman, and J. Terning, Phys. Rev. D 70, 015012 (2004); G. Cacciapaglia, C. Csaki, G. Marandella, and J. Terning, Phys. Rev. D 75, 015003 (2007); C. Csaki and D. Curtin, Phys. Rev. D 80, 015027 (2009).

[6] A. Birkedal, K. Matchev, and M. Perelstein, Phys. Rev. Lett. 94, 191803 (2005); H. J. He et al., Phys. Rev. D 78, 031701 (2008); T. Ohl and C. Speckner, Phys. Rev. D 78, 095008 (2008); O. Cata, G. Isidori, and J. F. Kamenik, arXiv:0905.0490.

[7] A. Alves, O. J. P. Eboli, M. C. Gonzalez-Garcia, and J. K.
Mizukoshi, Phys. Rev. D 79, 035009 (2009).

[8] T. Stelzer and F. Long, Comput. Phys. Commun. 81, 357 (1994); F. Maltoni and T. Stelzer, J. High Energy Phys. 02 (2003) 027.

[9] J. Pumplin, D. R. Stump, J. Huston, H. L. Lai, P. Nadolsky, and W. K. Tung, J. High Energy Phys. 07 (2002) 012.

[10] D. E. Acosta et al. (CDF Collaboration), Phys. Rev. D 66, 012004 (2002); T. Aaltonen et al. (CDF Collaboration), Phys. Rev. D 79, 112002 (2009).

[11] A. Abulencia et al. (CDF Collaboration), Phys. Rev. D 75, 091101 (2007); V. M. Abazov et al. (D0 Collaboration), Phys. Rev. Lett. 100, 211803 (2008); M. P. Giordani (CDF and D0 Collaborations), Eur. Phys. J. C 33, s785 (2004); R. J. Hooper (D0 Collaboration), Int. J. Mod. Phys. A 20, 3277 (2005).

[12] D. Rainwater, Ph.D. thesis, University of Wisconsin, Madison [arXiv:hep-ph/9908378].

[13] V. Cavasinni, D. Costanzo, and I. Vivarelli, Report No. ATL-PHYS-2002-008; S. Asai et al., Eur. Phys. J. C 32, s19 (2004).

[14] K. Agashe et al., Phys. Rev. D 76, 115015 (2007).

[15] J. M. Campbell, E. Castaneda-Miranda, Y. Fang, N. Kauer, B. Mellado, and S. L. Wu, arXiv:0906.2500. 